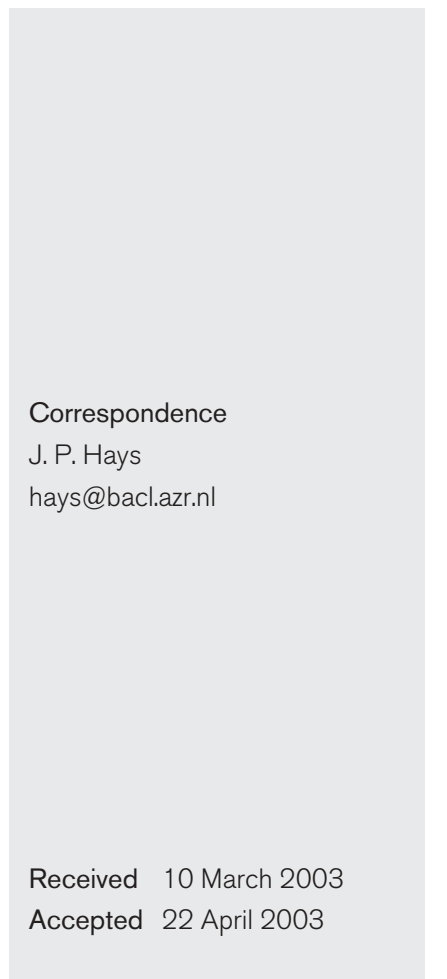

\title{
Changes in genetic types and population dynamics of Moraxella catarrhalis in hospitalized children are not associated with an exacerbation of existing disease
}

\author{
J. P. Hays, K. Eadie, C. M. Verduin, J. Hazelzet, H. Verbrugh and A. van Belkum
}

Department of Medical Microbiology and Infectious Diseases, Erasmus University Medical Center, Rotterdam (EMCR), Dr Molewaterplein 40, 3015 GD Rotterdam, The Netherlands

\begin{abstract}
Pulsed-field gel electrophoresis typing was performed on a retrospective set of 129 Moraxella catarrhalis isolates obtained over a 20 month period from 70 children admitted to, or presenting at, the Erasmus University Medical Center, Rotterdam, The Netherlands. The mean age of the children (at the end of the study) was 2.5 years, with a range of 6 months to 15 years. Fifty-one different $M$. catarrhalis types were isolated from the hospitalized children, with $31 \%(22 / 70)$ being infected with two particularly prevalent $M$. catarrhalis types. These two prevalent types also exhibited different protein profiles. The majority $(72 \% ; 16 / 22)$ of the children infected with these two predominant types had spent at least 1 week on two paediatric intensive care wards. No exacerbation of existing disease or new disease was observed in children who experienced $M$. catarrhalis type changes.
\end{abstract}

\section{INTRODUCTION}

Moraxella catarrhalis is often found as a commensal bacterium of humans, though the species has also been recognized as a major respiratory pathogen of both children and adults (Verduin et al., 2002). The organism is most often associated with the respiratory disease states of acute and chronic otitis media (Faden, 1995; Faden et al., 1997), sinusitis (Finegold et al., 2002) and acute exacerbations of chronic obstructive pulmonary disease (COPD) (Pfaller et al., 2001; Sethi \& Murphy, 2001). In common with several other respiratory pathogens, $M$. catarrhalis has the ability to adhere to bronchial epithelial cells, facilitate ciliotoxicity and ultimately damage pulmonary epithelia (van Alphen et al., 1995), all of which add to the pathogenic potential of this species.

Nosocomial pneumonia is the most frequently acquired infection in the intensive care unit (ICU) setting and the second most frequently acquired infection in hospitals overall (Lode et al., 2000). In ventilated children who contract nosocomial pneumonia, an attributable mortality of $27 \%$ may be observed (rising to $43 \%$ where Pseudomonas or Acinetobacter species are cultured) (Fagon et al., 1993). Reports of nosocomial infections due to M. catarrhalis have already been published (Cook et al., 1989; Denamur et al., 1989; Kasian et al., 1989; Morgan et al., 1992; Patterson et al., 1988; Richards et al., 1993), with Ikram et al. (1993) indicating that the average length of stay in hospital is

Abbreviation: PFGE, pulsed-field gel electrophoresis. considerably longer for children colonized with M. catarrhalis than for those not colonized and providing evidence that recolonization with different $M$. catarrhalis types occurs. Recently, it has been observed that the isolation of a new strain of Streptococcus pneumoniae, Haemophilus influenzae or M. catarrhalis is associated with a significantly increased risk of experiencing an exacerbation of COPD in adults (Sethi et al., 2002).

The aim of this study was to assess M. catarrhalis population diversity/dynamics retrospectively in hospitalized children and to determine whether $M$. catarrhalis type changes resulted in exacerbation of existing disease within this study population.

\section{METHODS}

Study population. M. catarrhalis isolates were collected retrospectively from 70 children admitted to, or presenting at, the Erasmus Medical Center during the study period of July 2000 to March 2002. Sixty-one of these 70 children had been admitted to diverse paediatric wards within the hospital (including neonatology, paediatric intensive care, children's surgery and children's health departments) for various periods of time. Nine children had attended out-patient clinics and had not been admitted to the Erasmus Medical Center between the beginning of the study (July 2000) and the date of isolation of the first positive culture. The children lived in geographically diverse regions within The Netherlands and suffered from unrelated disease states (e.g. tetralogy of Fallot, HIV-positive, Down's syndrome, etc). The mean age of children from which isolates were obtained was 2.5 years, with a range of 0.5 to 15 years. 
M. catarrhalis specimens. A total of 129 retrospectively cultured and stored M. catarrhalis isolates (which had been isolated from routine swabs/swabs taken from children presenting with overt signs of disease over a 20 month study period) were collected ready for typing. During the time period of the study, four or more $M$. catarrhalis culture positives were available from $10 \%(7 / 70)$ of the children, with only a single positive culture being available from $76 \%$ (53/70) of the children. M. catarrhalis-positive cultures came from 79 sputa, 38 bronchial aspirates, nine bronchial lavages, one eye swab, one nose swab and one drain tip.

M. catarrhalis typing. Pulsed-field gel electrophoresis (PFGE) was performed as detailed by Verduin et al. (2000). PFGE patterns were analysed using GelCompar software (Applied Maths) with band tolerance set at $2.5 \%$ after gel lanes had been normalized against a lambda DNA ladder. SDS-PAGE was performed using a discontinuous Laemmli buffer system (4\% stacking gel, $11 \%$ separating gel) and the Mini-PROTEAN 3 Cell electrophoresis kit (Bio-Rad). Eight M. catarrhalis isolates of PFGE type $\mathrm{E}$ or $\mathrm{M}$ (four from each group) were chosen at random for SDS-PAGE analysis.

Clinical data. Clinical data were collected from patients' clinical records, containing details of existing disease, dates and wards to which children were admitted, dates discharged from hospital, any treatment procedures (e.g. decanulation, extubation), any reports of exacerbation of disease (e.g. increased mucus, fever) and bacterial/viral investigations (e.g. bacterial culture, viral immunofluorescence).

\section{RESULTS AND DISCUSSION}

\section{Diversity in M. catarrhalis types}

During the 20 month study period (July 2000-March 2002), a total of 51 different types of $M$. catarrhalis were isolated from 70 children admitted to, or presenting at, the Erasmus Medical Center. Of these 51 types, four ( $8 \%$ ) were isolated from two distinct children each and two 'predominant' types (types E and M) were respectively isolated from 15 (21\%) and nine (13\%) children (Fig. 1). Fig. 2 shows the dendrogram generated when these 51 different $M$. catarrhalis types

Total number of children $=70$

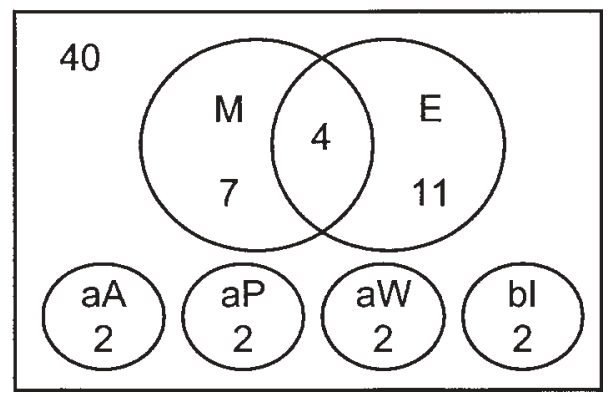

Fig. 1. Venn diagram showing the numbers of different children colonized with particular M. catarrhalis PFGE types. Letters represent PFGE types, with numbers of individual children infected with that PFGE type indicated. The number 40 represents the number of children in which only a single PFGE type was isolated during the study period. were compared; multiple isolates of the same type from the same child were removed in order to generate a more accurate view of the diversity of $M$. catarrhalis types present within the study population. A large number of individual $M$. catarrhalis PFGE types were observed within the study population of hospitalized children. This large number of types probably reflects the high degree of genetic diversity present within this species, a diversity that appears to be a feature of M. catarrhalis infection and colonization (Faden et al., 1994; Hays et al., 2003; Martinez et al., 1999) and which may present problems for vaccine design. However, despite this diversity, two distinct but closely related clusters of PFGE types (types E and M) could be observed within the dendrogram of isolates. Upon SDS-PAGE analysis of four randomly chosen isolates from groups $\mathrm{E}$ and $\mathrm{M}$, it was observed that individual isolates within the $\mathrm{E}$ and $\mathrm{M}$ clusters also shared similar protein profiles and that these protein profiles differed between the $\mathrm{E}$ and $\mathrm{M}$ clusters (Fig. 3). The presence/absence of a protein of approximately $40 \mathrm{kDa}$ was particularly noticeable. The inclusion of PFGE banding patterns obtained from four randomly selected complement-sensitive and complement-resistant isolates (acquired from Verduin et al., 2000) in the dendrogram shown in Fig. 2 indicated no grouping of complement-resistant or -sensitive PFGE banding patterns to one or other of the clusters $\mathrm{E} / \mathrm{M}$ or to any particular region of the dendrogram (data not shown).

\section{The hospital setting}

Examination of $M$. catarrhalis type data and hospital location indicated that diverse types could be found on all children's wards of the hospital. Moreover, though two dendrogram clusters of PFGE types could be observed, neither of these clusters was found to be $100 \%$ associated with children admitted to any particular ward. However, more-specific analysis showed an association between isolation of the predominant types $\mathrm{E}$ and $\mathrm{M}$ and admission of children for 1 week or more to two adjacent paediatric intensive care wards (Table 1). By comparing the numbers of children colonized with type $\mathrm{M}$ and admitted to paediatric intensive care wards with those colonized with type $\mathrm{M}$ and admitted to other wards, it was found that colonization by type $\mathrm{M}$ was associated statistically with the paediatric intensive care wards (2-tailed Fisher's exact test; $P<0 \cdot 01$ ), though colonization of children with type $\mathrm{M}$ was not absolutely dependent on a stay of more than 1 week in paediatric intensive care (2-tailed Fisher's exact test; $P=0 \cdot 067$ ). Of the four other $M$. catarrhalis types isolated from more than one child (Fig. 1), type aA was associated with two children admitted to the paediatric intensive care wards (where types $\mathrm{E}$ and $\mathrm{M}$ were predominant), type bI was isolated from two children who were admitted to the same neonatology ward and types aP and aW were isolated from children with no common link. It is possible that a larger study would indicate that other PFGE types are associated statistically with the paediatric intensive care setting or that colonization of children with the same $M$. catarrhalis type is not restricted 


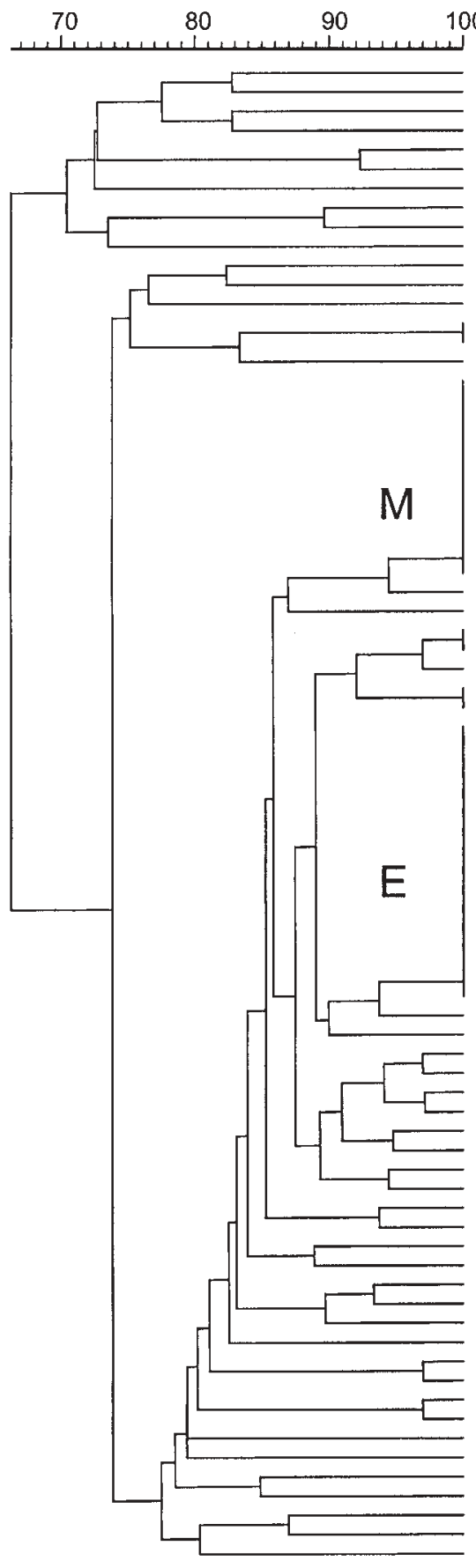

Patient

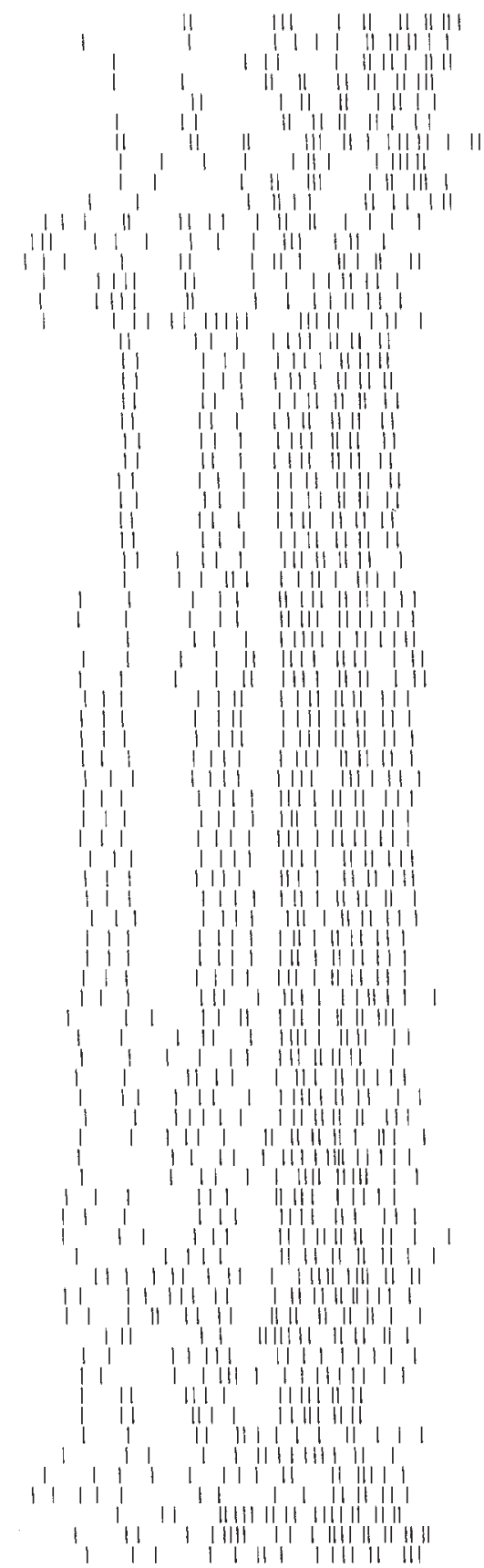

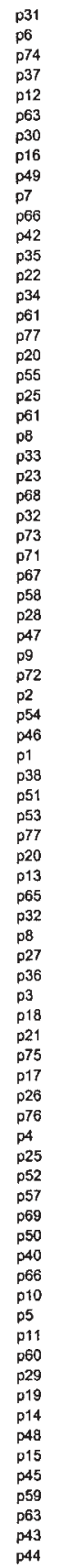

Fig. 2. Dendrogram showing PFGE patterns of M. catarrhalis isolates obtained from children admitted to or attending a hospital in the Netherlands. PFGE was performed and patterns were analysed as described in Methods. Letters $\mathrm{E}$ and $\mathrm{M}$ represent isolates with similar PFGE patterns that were subsequently grouped into $M$. catarrhalis types $E$ and $M$.

to the paediatric intensive care setting. It should also be noted that there might have been a natural bias towards the isolation of common types within the two paediatric intensive care wards. This results from the larger number of swabs taken regularly from children admitted to these two wards. However, this possible bias does not affect our results regarding the diversity and dynamics of $M$. catarrhalis types isolated from hospitalized children. We may merely have underestimated the extent of $M$. catarrhalis dynamic type changes within this environment.

\section{M. catarrhalis type switching}

Based on the results shown in Table 1, a timeline can be 


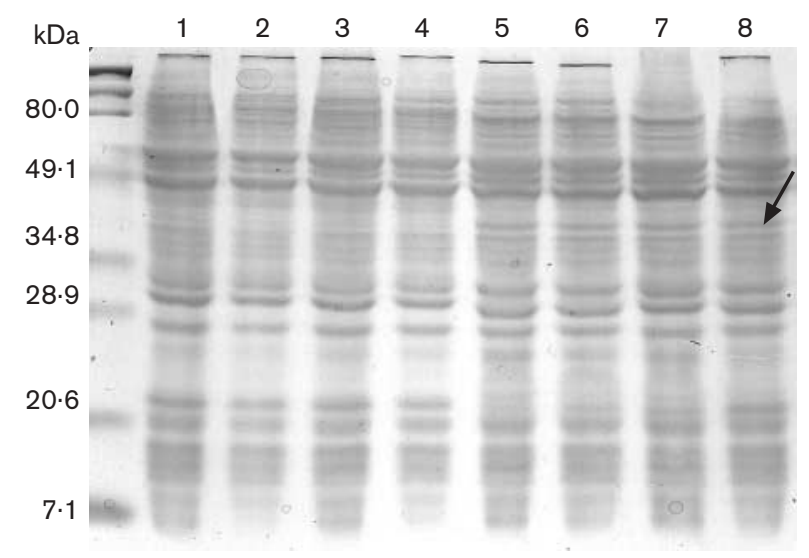

Fig. 3. SDS-PAGE protein profiles obtained using four randomly chosen isolates from each of $M$. catarrhalis types $M$ (lanes 1-4) and $E(5-8)$. The major difference in the protein band profiles of types $\mathrm{E}$ and $\mathrm{M}$ is indicated by the arrow.

drawn for children admitted to the two paediatric intensive care wards and colonized with types $\mathrm{E}$ and $\mathrm{M}$ during the course of the study (Fig. 4). Using this timeline, a dynamic relationship was observed between these two dominant types and an important time period (between February and April 2001), where type switching had occurred. The actual mechanism underlying this type switching is not fully understood, though three mechanisms are possible: (i) changes in the dominant type present within a population of organisms colonizing a single individual lead to isolation of different types from that individual over different time periods, (ii) a new (potentially more virulent) type is introduced into the hospital from the outside community and colonizes hospitalized individuals/health-care workers, e.g. during visiting hours, or (iii) transfer of types occurs within the hospital environment per se, e.g. from child to child or from health-care worker to child. Interestingly, isolates from child p77 showed switching between types $\mathrm{E}$ and $\mathrm{M}$ within the same month on several occasions (Fig. 4), possibly indicating that a mixed population of $M$. catarrhalis types may have colonized this child (repeated cycles of infection, clearance and reinfection with these two types seem unlikely, as the child had an intact immune system). However, with regard to community and hospital spread of types, of $37 \mathrm{M}$. catarrhalis isolates from children who had not been admitted to the two paediatric intensive care wards where types $\mathrm{E}$ and M were predominant, $14 \%$ (5/37) of children were colonized with $M$. catarrhalis type E, indicating that type E was circulating either within the community or within the whole of the hospital during the time-course of the study. In contrast, type $\mathrm{M}$ was only ever isolated from the paediatric intensive care wards, indicating that the spread of this type was probably occurring within these wards themselves. With reference to these data and the timeline of Fig. 4, it seems likely that child p77 may have been the index case for the spread of type $M$ within these paediatric intensive care wards. Certainly, the previous transmission of viruses from child to child and/or child to health-care worker in a neonatal and paediatric ICU has been documented (Gagneur et al., 2002a, b).

\section{Changes in M. catarrhalis type and clinical data}

Analysis of clinical data showed no obvious new treatment procedures taking place on the paediatric wards during the period of the study, and no other epidemic infectious agents (e.g. Mycoplasma pneumoniae, parainfluenzaviruses, etc.) had been isolated from the wards. Also, no link was found between changes in $M$. catarrhalis type and isolation in 'pure' culture of M. catarrhalis or changes in M. catarrhalis type and isolation in combination with other bacterial pathogens (e.g. $H$. influenzae, Pseudomonas aeruginosa, Staphylococcus aureus, etc.). No change in antibiotic-resistance profiles occurred after a change in M. catarrhalis type, with both types $\mathrm{E}$ and $\mathrm{M}$ being resistant to penicillin and ampicillin but sensitive to augmentin, ceftriaxon and tetracycline. During the 20 months of the study, several other infectious agents were isolated from one or more of the hospitalized children. These infectious agents included, among others, cytomegalovirus, parainfluenzavirus type 3 , enterovirus and respiratory syncytial virus. Interestingly, the clinical data showed that child p29 had been diagnosed with pneumonia whilst admitted to one of the paediatric intensive care wards. This

Table 1. M. catarrhalis PFGE types and lengths of stay for 70 children admitted to or attending a

Dutch hospital

PFGE types detected in individual children in each setting are listed according to their length of stay.

\begin{tabular}{|c|c|c|}
\hline \multirow[t]{2}{*}{ Wards } & \multicolumn{2}{|c|}{ Length of stay } \\
\hline & $<1$ week & $>1$ week \\
\hline Paediatric intensive care & $\mathrm{C}, \mathrm{aU}, \mathrm{Z}, \mathrm{aZ}, \mathrm{bA}, \mathrm{aR}, \mathrm{aA}$ & $\begin{array}{c}\text { E/M, aI, D, E, aW, E/M, M, aF/M, E, } \\
\text { aA, aT, J, F, E/M, E, bL, bK, E, E, } \\
\text { M, bB/M, bH/G, M, M, E/M }\end{array}$ \\
\hline Other & $\begin{array}{l}\text { E, aP, aE, bG, K, A, Y, P, E, bE, B, } \\
\quad \mathrm{aC}, \mathrm{E}, \mathrm{aG}, \mathrm{aS}, \mathrm{E}, \mathrm{aQ}, \mathrm{W}, \mathrm{aX}, \mathrm{aH}, \mathrm{H}\end{array}$ & $\begin{array}{l}\mathrm{E}, \mathrm{X}, \mathrm{aM}, \mathrm{aL}, \mathrm{aW}, \mathrm{bI}, \mathrm{bI}, \mathrm{bM}, \mathrm{I}, \mathrm{E}, \mathrm{aB}, \\
\mathrm{aN}, \mathrm{bJ}, \mathrm{bC} / \mathrm{aK}, \mathrm{N}, \mathrm{Ap}, \mathrm{M}\end{array}$ \\
\hline
\end{tabular}




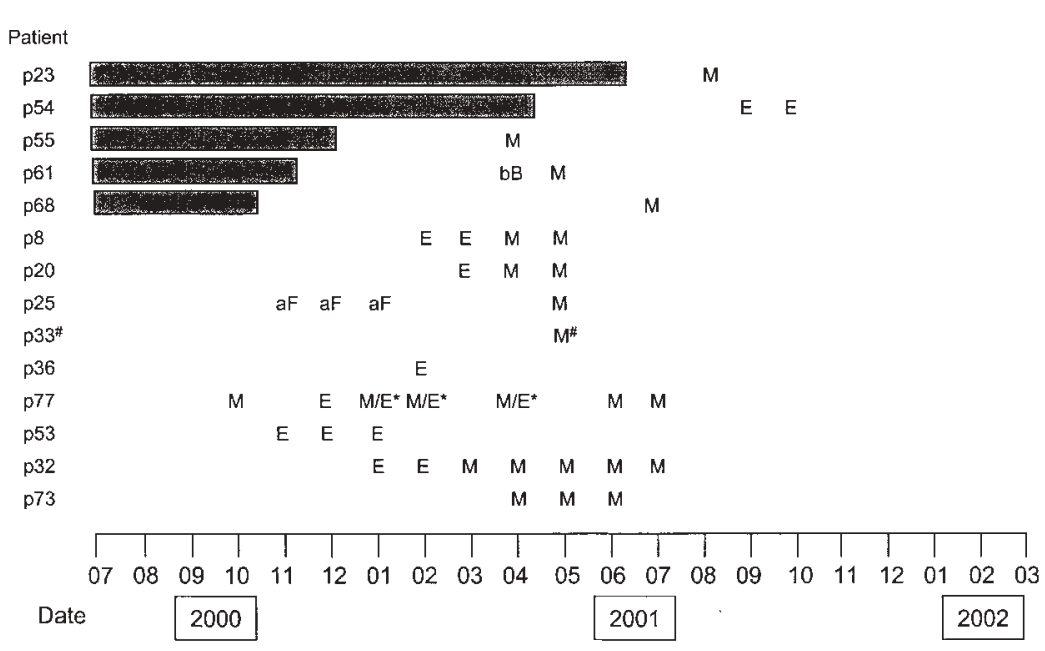

Fig. 4. Timeline showing dynamic population changes observed between $M$. catarrhalis PFGE types $E$ and $M$ in hospitalized children on two geographically linked paediatric intensive care wards. Filled rectangles indicate periods before the birth of a child. Letters are PFGE types. Asterisks $\left({ }^{*}\right)$ indicate that both PFGE types $E$ and $M$ were isolated during these months. Patient p33 (\#) had previously been admitted to paediatric intensive care but, at the time of M. catarrhalis culture, was present on an unrelated child health ward (none of the other children had been admitted to this particular ward).

child had recently undergone surgery to repair patent ductus arteriosus of the heart and both M. catarrhalis and Staphylococcus aureus were isolated from consecutive sputum samples. However, the M. catarrhalis cultured from this child was not PFGE type E or M (Fig. 2) and the child was discharged in good health approximately 3 weeks later. Adults with COPD have previously been shown to develop new serum IgG and new mucosal IgM to $M$. catarrhalis after exacerbations (Bakri et al., 2002). Moreover, a recent study has indicated an association between the isolation of a new strain of a particular bacterial species (including M. catarrhalis) and exacerbation of COPD in adults (Sethi et al., 2002). However, our own studies of available clinical records indicated no link between the acquisition of a new $M$. catarrhalis type and subsequent exacerbation of existing disease (or development of new disease) within hospitalized children.

These results indicate that dynamic changes in nosocomial M. catarrhalis populations occur in individual hospitalized children over time and that these changes are set against an already genetically diverse background of $M$. catarrhalis types. In contrast to recent results, no relationship could be determined between changes in $M$. catarrhalis type and exacerbation of disease. However, more detailed and rigorously controlled studies would be useful in assessing the exact effect of M. catarrhalis type changes on child morbidity, as well as helping to elucidate the mechanism by which such population changes occur.

\section{ACKNOWLEDGEMENTS}

The authors would like to acknowledge the work of the bacteriology department of the Erasmus Medical Center for their help with initial isolation of $M$. catarrhalis isolates and Dr J. Nouwen for advice regarding statistical analysis of results.

\section{REFERENCES}

Bakri, F., Brauer, A. L., Sethi, S. \& Murphy, T. F. (2002). Systemic and mucosal antibody response to Moraxella catarrhalis after exacerbations of chronic obstructive pulmonary disease. J Infect Dis 185, 632-640.
Cook, P. P., Hecht, D. W. \& Snydman, D. R. (1989). Nosocomial Branhamella catarrhalis in a paediatric intensive care unit: risk factors for disease. J Hosp Infect 13, 299-307.

Denamur, E., Suermondt, G., Debroca, A., Defouilloy, C., Laurans, G., Muir, J. F. \& Orfila, J. (1989). Nosocomial pulmonary infections caused by Branhamella catarrhalis in intensive care units. Agressologie 30, 251253 (in French).

Faden, H. (1995). Comparison of the local immune response to nontypable Haemophilus influenzae (nHI) and Moraxella catarrhalis (MC) during otitis media. Adv Exp Med Biol 371B, 733-736.

Faden, H., Harabuchi, Y. \& Hong, J. J. (1994). Epidemiology of Moraxella catarrhalis in children during the first 2 years of life: relationship to otitis media. J Infect Dis 169, 1312-1317.

Faden, H., Duffy, L., Wasielewski, R., Wolf, J., Krystofik, D. \& Tung, Y. (1997). Relationship between nasopharyngeal colonization and the development of otitis media in children. Tonawanda/Williamsville Pediatrics. J Infect Dis 175, 1440-1445.

Fagon, J. Y., Chastre, J., Hance, A. J., Montravers, P., Novara, A. \& Gibert, C. (1993). Nosocomial pneumonia in ventilated patients: a cohort study evaluating attributable mortality and hospital stay. Am J Med 94, 281-288.

Finegold, S. M., Flynn, M. J., Rose, F. V., Jousimies-Somer, H., Jakielaszek, C., McTeague, M., Wexler, H. M., Berkowitz, E. \& Wynne, B. (2002). Bacteriologic findings associated with chronic bacterial maxillary sinusitis in adults. Clin Infect Dis 35, 428-433.

Gagneur, A., Legrand, M. C., Picard, B., Baron, R., Talbot, P. J., de Parscau, L. \& Sizun, J. (2002a). Nosocomial infections due to human coronaviruses in the newborn. Arch Pediatr 9, 61-69 (in French).

Gagneur, A., Sizun, J., Vallet, S., Legr, M. C., Picard, B. \& Talbot, P. J. (2002b). Coronavirus-related nosocomial viral respiratory infections in a neonatal and paediatric intensive care unit: a prospective study. J Hosp Infect 51, 59-64.

Hays, J. P., van der Schee, C., Loogman, A., Eadie, K., Verduin, C., Faden, H., Verbrugh, H. \& van Belkum, A. (2003). Total genome polymorphism and low frequency of intra-genomic variation in the uspA1 and uspA2 genes of Moraxella catarrhalis in otitis prone and nonprone children up to 2 years of age. Consequences for vaccine design? Vaccine 21, 1118-1124.

Ikram, R. B., Nixon, M., Aitken, J. \& Wells, E. (1993). A prospective study of isolation of Moraxella catarrhalis in a hospital during the winter months. J Hosp Infect 25, 7-14. 
Kasian, G. F., Shafran, S. D. \& Shyleyko, E. M. (1989). Branhamella catarrhalis bronchopulmonary isolates in PICU patients. Pediatr Pulmonol 7, 128-132.

Lode, H., Raffenberg, M., Erbes, R., Geerdes-Fenge, H. \& Mauch, H. (2000). Nosocomial pneumonia: epidemiology, pathogenesis, diagnosis, treatment and prevention. Curr Opin Infect Dis 13, 377-384

Martinez, G., Ahmed, K., Zheng, C. H., Watanabe, K., Oishi, K. \& Nagatake, T. (1999). DNA restriction patterns produced by pulsed-field gel electrophoresis in Moraxella catarrhalis isolated from different geographical areas. Epidemiol Infect 122, 417-422.

Morgan, M. G., McKenzie, H., Enright, M. C., Bain, M. \& Emmanuel, F. X. (1992). Use of molecular methods to characterize Moraxella catarrhalis strains in a suspected outbreak of nosocomial infection. Eur J Clin Microbiol Infect Dis 11, 305-312.

Patterson, T. F., Patterson, J. E., Masecar, B. L., Barden, G. E., Hierholzer, W. J., Jr \& Zervos, M. J. (1988). A nosocomial outbreak of Branhamella catarrhalis confirmed by restriction endonuclease analysis. J Infect Dis 157, 996-1001.

Pfaller, M. A., Ehrhardt, A. F. \& Jones, R. N. (2001). Frequency of pathogen occurrence and antimicrobial susceptibility among community-acquired respiratory tract infections in the respiratory surveillance program study: microbiology from the medical office practice environment. Am J Med 111 (Suppl. 9A), 4S-12S.
Richards, S. J., Greening, A. P., Enright, M. C., Morgan, M. G. \& McKenzie, H. (1993). Outbreak of Moraxella catarrhalis in a respiratory unit. Thorax 48, 91-92.

Sethi, S. \& Murphy, T. F. (2001). Bacterial infection in chronic obstructive pulmonary disease in 2000: a state-of-the-art review. Clin Microbiol Rev 14, 336-363.

Sethi, S., Evans, N., Grant, B. J. \& Murphy, T. F. (2002). New strains of bacteria and exacerbations of chronic obstructive pulmonary disease. $N$ Engl J Med 347, 465-471.

van Alphen, L., Jansen, H. M. \& Dankert, J. (1995). Virulence factors in the colonization and persistence of bacteria in the airways. Am J Respir Crit Care Med 151, 2094-2099.

Verduin, C. M., Kools-Sijmons, M., van der Plas, J., Vlooswijk, J., Tromp, M., van Dijk, H., Banks, J., Verbrugh, H. \& van Belkum, A. (2000). Complement-resistant Moraxella catarrhalis forms a genetically distinct lineage within the species. FEMS Microbiol Lett 184, 1-8.

Verduin, C. M., Hol, C., Fleer, A., van Dijk, H. \& van Belkum, A. (2002). Moraxella catarrhalis: from emerging to established pathogen. Clin Microbiol Rev 15, 125-144. 